\title{
Intra-Operative Damage Control Maneuvers to Reduce Abdominal Compartment Syndrome
}

\author{
Andrew Maloney ${ }^{1}$ - Paula Ferrada ${ }^{1}$ \\ Published online: 11 October 2016 \\ (C) Springer International Publishing AG 2016
}

\begin{abstract}
Purpose of Review Abdominal compartment syndrome (ACS) is defined as a sustained intra-abdominal pressure (IAP) greater than $20 \mathrm{mmHg}$ that is associated with the development of new organ dysfunction or failure.

Recent Findings Resuscitation to euvolemia continues to be a challenge, and in patients at risk of developing ACS, this is critical since fluid overload plays a major role in this development.

Summary The following article addressed maneuvers that can result in decrease incidence of ACS.
\end{abstract}

Keywords Abdominal compartment syndrome .

Intra-operative resuscitation $\cdot$ Euvolemic resuscitation

\section{Introduction}

Fluid resuscitation to euvolemia is one of the main paths to avoid abdominal compartment syndrome on the surgical patient [1]. To understand the swinging pendulum of the surgical literature in this matter, we have summarized the historical development of volume resuscitation.

The use of normal saline as an effective lifesaving therapy was first described when treating cholera patients in the $1800 \mathrm{~s}$ and evolved into the accepted therapy for other sources of

This article is part of the Topical Collection on Abdominal Compartment Syndrome in Trauma

Paula Ferrada

paula.ferrada@vcuhealth.org

1 VCU Surgery Trauma, Critical Care and Emergency Surgery, West Hospital, 15th Floor, East Wing, 1200 E. Broad St, PO Box 980454, Richmond, VA 23298-0454, USA hypovolemia including blood loss [2]. Later on, Carl John Wiggers performed meticulous experimentation, and in 1921, he was able to describe the relationship of the electrical cardiac cycle and cardiac valves, along with atrial, ventricular, and aortic pressures and their relationship with volume. The diagram of his cardiac cycle is still used in physiology courses today [3]. He also developed an experimental model of shock where dogs were subjected to hemorrhage down to differing systolic pressures [4]. A sea change in resuscitation principles was born out of basic science research performed by Tom Shires in Dallas, TX, during the late 1950s [5] He again used the Wiggers experiments, bleeding dogs according to protocols and experimenting with replacing shed blood and adding varying crystalloid and colloid solutions. His results showed a survival advantage in dogs resuscitated with shed blood plus $5 \%$ body weight lactated Ringer's.

Vietnam was a seminal conflict in terms of advancements in military medicine. The advent of rapid air ambulance evacuation of casualties resulted in critically injured patients reaching the field hospital before exsanguination, and the liberal crystalloid resuscitation practices borne out by Dr. Shires' research resulted in the first description of adult respiratory distress syndrome, initially titled "Da Nang Lung" due to a military hospital in the area. These practices were brought back to the civilian populations after the war, and in American trauma centers during the 1980s, it was routine for trauma patients to receive large bore central venous access and 20-30 L of crystalloid resuscitation in the first $24 \mathrm{~h}$ after injury. Adult respiratory distress syndrome (ARDS) and abdominal compartment syndrome were common maladies as a result $[6 \bullet \bullet]$.

The consequences of overzealous crystalloid resuscitation were anecdotally known throughout trauma centers in the 1980s, but it was Mattox's seminal work on delayed fluid resuscitation in 1994 that introduced new ideas regarding 
low-volume resuscitation $[6 \bullet \bullet, 7]$. This study enrolled all hypotensive-penetrating torso traumas to standard vs. delayed crystalloid fluid resuscitation and was blessed with a robust recruitment secondary to an ongoing war for control of the Houston cocaine market at the time. Patients were randomized to receive either a standard 2-L crystalloid resuscitation vs. withholding resuscitation until in the operating room. A statistically significant difference in hospital stays and overall survival benefitted the delayed resuscitation group, and another change in ideas towards crystalloid resuscitation was afoot.

Following Mattox findings, a prospective randomized trial performed at the R. Adams Cowley Shock-Trauma Center in 2002 was published [8]. In this trial, hypotensive trauma patients were randomized to a blood pressure goal of $70 \mathrm{vs}$. $100 \mathrm{mmHg}$ systolic pressure and found no difference in mortality. In contradistinction to Mattox's study, blunt trauma as well as penetrating was included.

The concepts of permissive hypotension and avoiding crystalloid resuscitation would be brought to the forefront during another American conflict in Iraq and Afghanistan. Rapid transport to forward field hospitals afforded a quicker control of surgical bleeding than the Vietnam experience, and again logistic difficulties prevented a conventional system of storing donated blood and transporting it to a site of need. The concept of the walking blood bank was revisited over 50 years after WWII and was a resounding success. Tertiary-referring hospitals in Germany and the continental USA witnessed massively transfused patients arriving warm, hemodynamically stable, and without the expected coagulopathy or acidosis. Retrospective studies [9] showed a statistically significant increase in survival for the patients resuscitated with warm fresh whole blood (WFWB) compared to transfusions based on blood components.

Unfortunately for the civilian population, a walking blood bank is a near impossibility and civilian trauma centers set out to approximate WFWB for their own hemorrhaging patients. Multiple studies in the early and late 2000s evaluated patient outcomes based on the type of resuscitation that massively transfused trauma patients received. Packed red blood cell (PRBC):fresh frozen plasma (FFP) ratios were used as a surrogate marker of the new concept of "hemostatic" resuscitation and noted to correlate with survival [10]. An obvious bias towards the massively hemorrhaging moribund patient who does not survive long enough to activate the blood bank would favor lower RBC:FFP ratios was accounted for by excluding those who died within $1 \mathrm{~h}$ of arrival. Results strongly favored low PRBC:FFP ratios in multiple trials and further research into RPBC:platelet ratios continued to show improvements in outcomes.

As of the late 2000s, the state of the art resuscitation in American civilian trauma centers revolved around the creation of massive transfusion protocols (MTP) [11]. However, patients continued to receive high volumes of crystalloid resuscitation, albeit less than the days before Mattox's study where trauma patients were expected to suffer from ARDS. It was a natural progression to attempt to combine low ratio PRBC:FFP resuscitation that approximated whole blood with a low-volume crystalloid policy that minimized the deleterious effects of crystalloids $[12 \bullet \bullet, 13 \bullet \cdot$. Studies in this area are ongoing but single center studies are showing statistically significant decreases in mortality of massively transfused patients receiving low volumes of crystalloid. Multi-institutional analyses $[12 \bullet \cdot 13 \bullet \cdot]$ of these studies are showing odds ratio reductions for multiple sequelae of the pro-inflammatory state such as surgical site infection, urinary tract infection, bacteremia, sepsis, acute respiratory distress syndrome, acute renal failure, and ventilator associated pneumonia [14•].

In summary, thus far, there is no perfect fluid, nor a secret formula to resuscitate patients to avoid complications, other than maintaining euvolemia. In order to achieve this goal, early bleeding control and low volume resuscitation using an armamentarium of hemodynamic monitoring tools is desirable.

\section{Principles of Management}

- Early bleeding control: This is a point that deserves emphasis regardless of the primary disease process of the patient. Achieving early control of bleeding in trauma or in emergency general surgery is desirable and would avoid problems with coagulopathy and other physiological consequences. Until the bleeding is controlled, maintaining low systolic blood pressure is necessary to avoid unnecessary bleeding.

- Maintaining euvolemia: This is a pivotal point of avoiding complications from intra-operative resuscitation. This becomes even more important when resuscitating elderly patients with comorbidities. If the patient is in the operating room secondary to an emergency general surgery problem, a preoperative estimation of cardiac function is desirable. Pulmonary artery catheters have fallen out of favor since the report from the New England Journal of Medicine of increasing pulmonary emboli incidence and not improving survival; however, in elderly patients with known pulmonary hypertension, this can be a valuable tool [15]. Trans-thoracic echo pre, intra-, and post operatively can provide with pivotal information in cardiac function and preload. In the operating room, and especially of the chest is not accessible trans-esophageal echocardiogram can be used for the same purpose [16•, 17-22]. Constant communication with an anesthesia is essential in avoiding over or under resuscitation which is associated with respiratory and renal complications, respectively [23]. If the patient is bleeding, then transfusing blood and products is required, and resuscitation with colloid solutions may provide an advantage in vasopressor use and ventilator days [24]. Fluid overload can contribute to 
bowel edema and possibly to future leaks, as well as being associated with soft tissue flap loss [25]. Stroke volume variation (SVV) can also be used as a goal of resuscitation in the intubated patient using an SVV that exceeds $13 \%$ as a parameter to indicate volume responsiveness [25].

- Recommendations:

- Consider a baseline measurement of the patient's cardiac function either with formal echocardiogram or surgeon performed echocardiogram in the urgent setting if possible.

- In the operating room, maintain close communication with anesthesia regarding intra-operative bleeding and need for blood or blood products transfusion attempting a $1: 1: 1$ ratio.

- Crystalloid infusion should be targeted to achieve the goal of euvolemia.

- Intra-operative monitors to aid in euvolemic resuscitation include SVV monitoring, trans-thoracic echocardiogram and trans-esophageal to evaluate cardiac function and volume status.

\section{Conclusions}

Goal-directed volume resuscitation to euvolemia has been shown to be the best approach for surgical patients. Choosing tools to manage euvolemia, depending on the resources available in each clinical center as well as constant communication with anesthesia, are pivotal issues to improve patient outcomes.

\section{Compliance with Ethical Standards}

Conflict of Interest Drs. Maloney and Ferrada declare no conflicts of interest.

Human and Animal Rights and Informed Consent This article does not contain any studies with human or animal subjects performed by any of the authors.

\section{References}

Papers of particular interest, published recently, have been highlighted as:

- Of importance

• Of major importance

1. Roberts DJ, Ball CG, Kirkpatrick AW. Increased pressure within the abdominal compartment: intra-abdominal hypertension and the abdominal compartment syndrome. Curr Opin Crit Care. 2016;22:174-85.
2. Moon JB. Sir William Brooke O'Shaughnessy — the foundations of fluid therapy and the Indian Telegraph Service. N Engl J Med. 1967;276(5):283-4.

3. Mitchell JR, Wang JJ. Expanding application of the Wiggers diagram to teach cardiovascular physiology. Adv Physiol Educ. 2014;38(2):170-5.

4. Edwards MR, Mythen MG. Fluid therapy in critical illness. Extreme Physiol Med. 2014;3:16.

5. Carrico CJ et al. Extracellular fluid volume replacement in hemorrhagic shock. Surg Forum. 1963;14:10-2.

6.• Rotondo MF et al. 'Damage control': an approach for improved survival in exsanguinating penetrating abdominal injury. $\mathrm{J}$ Trauma. 1993;35(3):375-82. discussion 382-3. A seminal work in the trauma literature showing increased survival for massively injuried patients treated with staged laparotomy.

7. Bickell WH et al. Immediate versus delayed fluid resuscitation for hypotensive patients with penetrating torso injuries. N Engl J Med. 1994;331(17):1105-9.

8. Dutton RP, Mackenzie CF, Scalea TM. Hypotensive resuscitation during active hemorrhage: impact on in-hospital mortality. J Trauma. 2002;52(6):1141-6.

9. Spinella PC et al. Warm fresh whole blood is independently associated with improved survival for patients with combat-related traumatic injuries. J Trauma. 2009;66(4 Suppl):S69-76.

10. Teixeira PG et al. Impact of plasma transfusion in massively transfused trauma patients. J Trauma. 2009;66(3): 693-7.

11. Dente CJ et al. Improvements in early mortality and coagulopathy are sustained better in patients with blunt trauma after institution of a massive transfusion protocol in a civilian level I trauma center. J Trauma. 2009;66(6):1616-24.

12.• Duchesne JC et al. Low-volume resuscitation for severe intraoperative hemorrhage: a step in the right direction. Am Surg. 2012;78(9):936-41. A case control study comparing low volume resuscitation with traditional resuscitation in massively bleeding patients, showing increased survival and decreased ICU length of stay in low volume hemostatic resuscitated patients.

13.・ Duchesne JC et al. Diluting the benefits of hemostatic resuscitation: a multi-institutional analysis. J Trauma Acute Care Surg. 2013;75(1):76-82. Multi-institutional analysis investigating high volume crystalloid and high ratio hemostatic resuscitation. Crystalloid use was associated with increased infectious and organ failure complications, the ratio of hemostatic resuscitation was not.

14. Guidry $\mathrm{C}$ et al. Initial assessment on the impact of crystalloids versus colloids during damage control resuscitation. J Surg Res. 2013;185(1):294-9. A single center matched case control study among bleeding trauma patients treated with high ratio hemostatic resuscitation. Higher amounts of crystalloid use were associated with higher mortality.

15. Sandham JD et al. A randomized, controlled trial of the use of pulmonary-artery catheters in high-risk surgical patients. N Engl J Med. 2003;348(1):5-14.

16. Ferrada, P., Image-based resuscitation of the hypotensive patient with cardiac ultrasound: an evidence-based review. J Trauma Acute Care Surg. 2015. An excellent review and instructive article regarding point of care cardiac ultrasound in the critically ill patient.

17. Ferrada $\mathrm{P}$ et al. Limited transthoracic echocardiogram: so easy any trauma attending can do it. J Trauma. 2011;71(5):1327-31. discussion 1331-2.

18. Ferrada $\mathrm{P}$ et al. Qualitative assessment of the inferior vena cava: useful tool for the evaluation of fluid status in critically ill patients. Am Surg. 2012;78(4):468-70. 
19. Ferrada $\mathrm{P}$ et al. Findings of a randomized controlled trial using limited transthoracic echocardiogram (LTTE) as a hemodynamic monitoring tool in the trauma bay. J Trauma Acute Care Surg. 2014;76(1):31-7. discussion 37-8.

20. Ferrada $\mathrm{P}$ et al. Transthoracic focused rapid echocardiographic examination: real-time evaluation of fluid status in critically ill trauma patients. J Trauma. 2011;70(1):56-62. discussion 62-4.

21. Ferrada $\mathrm{P}$ et al. A, B, C, D, echo: limited transthoracic echocardiogram is a useful tool to guide therapy for hypotension in the trauma bay - a pilot study. J Trauma Acute Care Surg. 2013;74(1):220-3.
22. Ferrada $\mathrm{P}$ et al. Use of limited transthoracic echocardiography in patients with traumatic cardiac arrest decreases the rate of nontherapeutic thoracotomy and hospital costs. J Ultrasound Med. 2014;33(10):1829-32.

23. Mason, S. et al., Hold the pendulum: rates of acute kidney injury are increased in patients who receive resuscitation volumes less than predicted by the Parkland equation. Ann Surg, 2016. Ahead of print.

24. Arunachalam $L$ et al. Colloid versus crystalloid fluid therapy in surgical patients. Br J Surg. 2015;102(3):145-7.

25. Funk D et al. Goal-directed fluid therapy for microvascular free flap reconstruction following mastectomy: a pilot study. Plast Surg (Oakv). 2015;23(4):231-4. 